CORRECTIONS

\title{
Author's reply to Girling
}

The word "not" was incorrectly introduced during editing to the third sentence in the second paragraph of this Letter $(B M J$ 2014;348:g4152, doi:10.1136/bmj.g4152), written by Heather Angus-Leppan. The sentence should have read, "Most presentations are antenatal and preterm, so women may present to non-maternity staff."

Cite this as: BMJ 2014;348:g4314

๑ BMJ Publishing Group Ltd 2014 